\title{
Balanced gain and loss in Bose-Einstein condensates without $\mathcal{P} \mathcal{T}$ symmetry
}

\author{
Philipp Lunt, Daniel Haag, ${ }^{*}$ Dennis Dast, Holger Cartarius, and Günter Wunner \\ Institut für Theoretische Physik 1, Universität Stuttgart, 70550 Stuttgart, Germany
}

(Dated: September 22, 2017)

\begin{abstract}
Balanced gain and loss renders the mean-field description of Bose-Einstein condensates $\mathcal{P} \mathcal{T}$ symmetric. However, any experimental realization has to deal with unbalancing in the gain and loss contributions breaking the $\mathcal{P} \mathcal{T}$ symmetry. We will show that such an asymmetry does not necessarily lead to a system without a stable mean-field ground state. Indeed, by exploiting the nonlinear properties of the condensate, a small asymmetry can stabilize the system even further due to a self-regulation of the particle number.
\end{abstract}

\section{INTRODUCTION}

Since the discovery of real eigenvalues in a nonHermitian $\mathcal{P} \mathcal{T}$-symmetric Hamiltonian by Bender and Boettcher in 1998 [1] a lot of work was put into replacing the usual concept of Hermitian quantum mechanics with the more general condition of $\mathcal{P} \mathcal{T}$ symmetry [2-4]. In the course of the search for experimental realizations, the attention shifted to optical systems, where $\mathcal{P} \mathcal{T}$ symmetry is accomplished by a positive and negative imaginary refractive index that in the equations effectively models a gain and loss of the field strength [5-9].

In fact the first realizations succeeded in two coupled optical waveguides. In the first experiment two different absorption strengths were used to create passive $\mathcal{P} \mathcal{T}$ symmetry [10], whereas in a subsequent realization one waveguide was actively pumped to amplify the field strength [11]. This showed that while the original concepts of $\mathcal{P} \mathcal{T}$ symmetry focused on fundamental changes in the nature of quantum mechanics, its first realizations succeeded in an effective mean-field description, which again attracted further theoretical and experimental efforts $[12,13]$. Another approach towards a realization lies in purely electronic frameworks $[14,15]$.

With the success of $\mathcal{P} \mathcal{T}$ symmetry in these mean-field systems in mind it is quite comprehensible that BoseEinstein condensates should also qualify for a realization [6]. In this many-particle system, the in- and outcoupling acts directly on the particle density, increasing or decreasing the number of particles. This interpretation of particle loss and gain recently lead to the first real quantum simulation of a $\mathcal{P} \mathcal{T}$-symmetric system using a ${ }^{6} \mathrm{Li}$ Fermi gas [16]. Numerical calculations in spatially extended potentials confirm that condensates are in principle able to provide all the effects known from linear optical realizations [17-21]. Proposals for an experimental realization in analogy to two optical waveguides have been made. They include embedding a double-well system in a longer chain of wells with time-dependent coupling parameters [22], and the description of two separate condensates exchanging their particles [23]. Furthermore, particle gain and loss can be realized by coupling particles

\footnotetext{
*daniel.haag@itp1.uni-stuttgart.de
}

into and out from the surrounding environment. Both processes have already been realized experimentally: Outcoupling by a focused electron beam [24] and in-coupling by letting atoms fall into the condensate from a second condensate [25].

In real systems a perfect control of the in- and outcoupling of particles is not possible. Therefore, asymmetries in the imaginary potential have to be expected, i.e., the system is not exactly $\mathcal{P} \mathcal{T}$ symmetric. Even though $\mathcal{P} \mathcal{T}$ symmetry is neither a necessary nor a sufficient condition for real eigenvalues [4] and its typical properties are also found in other systems [26, 27], it is not to be expected that such a perturbation leaves the stationary states intact. However, we will show that by increasing the in- and out-coupling parameter to a specific strength, one can restore a single real eigenvalue.

This paper is organized as follows. We start with a two-mode approximation, analyzing its eigenvalues and stability in Sec. II. Its dynamical properties and a comparison with the $\mathcal{P} \mathcal{T}$-symmetric double-well system are investigated in Sec. III. Afterwards, the discussion is extended to a spatially extended potential in Sec. IV.

\section{STATIONARY SOLUTIONS}

A Bose-Einstein condensate allowing for an asymmetry in the gain and loss of particles can be described by the Hamiltonian

$$
H=\left(\begin{array}{cc}
\mathrm{i} \gamma\left(1+a_{I}\right) & -1 \\
-1 & -\mathrm{i} \gamma\left(1-a_{I}\right)
\end{array}\right)
$$

where $a_{I} \in \mathbb{R}$ is the asymmetry between gain and loss and $\gamma$ is the dimensionless overall strength of the in- and out-coupling. The relative particle loss in the second well reads $2 \gamma / \tau$, where the timescale $\tau$ is fixed by the size and shape of a trapping potential. Using the doublewell experiment of [28] as an example, an approximate timescale of $\tau \approx 30 \mathrm{~ms}$ is found. Comparing this timescale to the losses realized in [29] shows, that such particle losses are well within experimental possibilities.

For $a_{I}=0$, the $\mathcal{P} \mathcal{T}$-symmetric two-mode model [17, 19, 30] is restored, where in the first well particles are injected into the system and particles are removed from the second well. In this system the ground and first excited state 
are $\mathcal{P} \mathcal{T}$ symmetric and have real eigenvalues up to an exceptional point, at which eigenstates and eigenvalues coalesce and vanish. From this point two $\mathcal{P} \mathcal{T}$-broken states with complex conjugate eigenvalues emerge.

This behavior changes drastically for $a_{I} \neq 0$. While the eigenvectors are not influenced by the asymmetry in the imaginary part, the eigenvalues of the Hamiltonian (1) now read

$$
\mu_{ \pm}=\mathrm{i} \gamma a_{I} \pm \sqrt{1-\gamma^{2}}
$$

Even though the second part of this equation shows the described behavior from the $\mathcal{P} \mathcal{T}$-symmetric case, the asymmetry $a_{I}$ generates a purely imaginary shift rendering both eigenvalues complex, i.e., they are no longer real stationary solutions of the Schrödinger equation.

One can easily check that one of the eigenvalues reaches $\operatorname{Im} \mu=0$ at a specific parameter value $\gamma_{0}=\sqrt{1 /\left(1-a_{I}^{2}\right)}$, thus, the corresponding eigenvector becomes stationary. We stress that at this intersection point $\gamma_{0}$ the other eigenstate of the two-mode system has the eigenvalue $\mu=2 \mathrm{i} a_{I}$ which means that it grows exponentially for $a_{I}>0$, for which the particle gain is stronger, and decays for $a_{I}<0$. The actual eigenstate is therefore stable only if an asymmetry is chosen in such a way that the particle loss is stronger than the gain. It can be shown that this requirement is, in fact, mandatory for spatially extended systems, since a stronger particle gain would enhance any high-energy perturbations that are equally strong in both wells.

However, a major problem remains. The actual stationary state exists only at one specific parameter $\gamma_{0}$ for a given asymmetry $a_{I}$. This leaves the experimental realization with the same problem as before since a small deviation from the desired gain or loss of particles could force the particle number to grow exponentially. However, the problem can be overcome by the particle-particle interactions present in a Bose-Einstein condensate if they are manufactured in such a way that a growth or decay in the particle number stabilizes the state.

Introducing a nonlinear contact interaction term leads to the two-mode Gross-Pitaevskii equation,

$$
\sum_{j=1}^{2} H_{i j} \psi_{j}+U\left|\psi_{i}\right|^{2} \psi_{i}=\mu \psi_{i}
$$

where $U$ specifies the strength of the interaction, which can take values from 0 to 2000 [31]. Such a contribution renders the spectrum dependent on the norm of the wave function. Thus, the parameter $\gamma$, for which the stationary state is found, changes with a growth or decay of the wave function. In the $\mathcal{P} \mathcal{T}$-symmetric case, $a_{I}=0$, analytical solutions were given by Graefe et al. [30]. With $U>0$, the $\mathcal{P} \mathcal{T}$-broken states no longer bifurcate at $\gamma=1$, at which the $\mathcal{P} \mathcal{T}$-symmetric states vanish. Instead, they emerge at an earlier point, $\gamma=\sqrt{1-U^{2} / 4}$ from the excited state. It is a solid hypothesis that a similar effect can be expected for $a_{I}<0$, where alongside the $\mathcal{P} \mathcal{T}$-broken
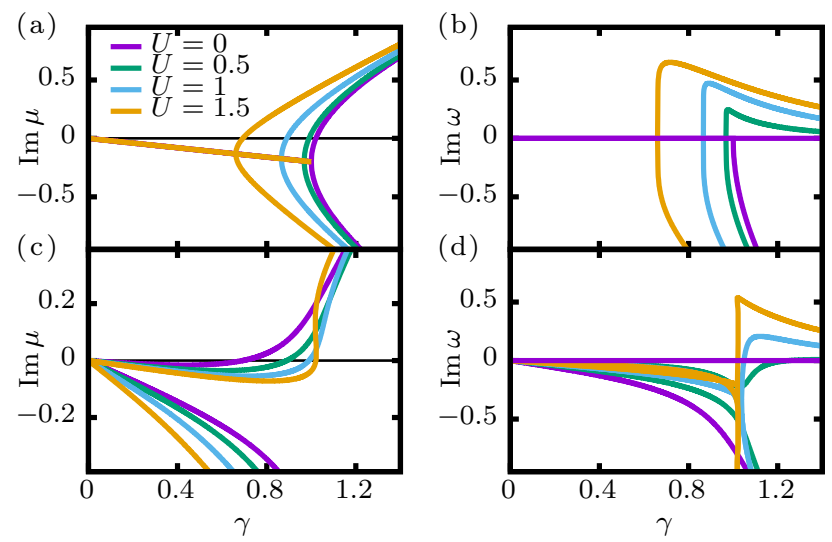

Figure 1. The imaginary part of the nonlinear spectrum (a) of the asymmetric double well (1) with $a_{I}=-0.2$. For stronger nonlinearity parameters $U$ the upper branches intersects the axis $\operatorname{Im} \mu=0$ at lower parameters $\gamma$. The imaginary part of the Bogoliubov-de Gennes eigenvalues $\omega(\mathrm{b})$ are given for the upper branch, which contains the stationary state. In the nonlinear case this state is unstable. For the system described by Eq. (4) with $a_{R}=-0.15$ (c), i.e., the case of asymmetric on-site energies, this behavior changes. Stronger nonlinearity parameters $U$ now lead to upper branches that intersect the axis $\operatorname{Im} \mu=0$ at larger parameters $\gamma$. This holds up to $U \approx 1.5$. For even stronger nonlinearities, the intersection with the axis again moves to lower values of $\gamma$. The appropriate stability eigenvalues of the Bogoliubov-de Gennes equations (d) are now negative imaginary numbers, which shows that the stationary state is a dynamical attractor.

states, also the intersection point $\gamma_{0}$ moves to lower values. This is confirmed by Fig. 1(a), which shows the eigenvalue spectrum for the nonlinearity parameters $U=0,0.5,1$ and 1.5. In these calculations, the asymmetry $a_{I}=-0.2$ was used.

Not only is the aforementioned hypothesis confirmed but the results already show that the system cannot be stable: Consider an experimental system in which the non-Hermiticity or gain-loss parameter $\gamma$ is chosen larger than $\gamma_{0}$. The ground state with $\operatorname{Im} \mu>0$ is then growing instead of staying stationary. This effectively increases the nonlinearity and shifts the intersection point of $\gamma$, at which the true stationary state with $\operatorname{Im} \mu=0$ can be found, to even lower values. The distance between the chosen and the correct value of $\gamma$ increases, and thus the error is amplifying itself due to the nonlinearity.

This instability can also be shown numerically using the corresponding Bogoliubov-de Gennes equations [32]. The eigenvalues of this system of equations describe how small perturbations of the eigenstate behave. Positive imaginary parts show an unstable behavior, while negative imaginary parts characterize a dynamical attractor. These eigenvalues are shown in Fig. 1(b) for the $\mathcal{P} \mathcal{T}$-broken state that intersects with $\operatorname{Im} \mu=0$ at $\gamma_{0}$. In agreement with our prediction there is a positive imaginary part and the states are unstable. 
The influence of the nonlinearity in this simple model not only fails to stabilize the stationary state against small deviations from $\gamma_{0}$ but introduces an instability against norm oscillations. To get rid of this additional instability the opposite behavior is required: A stronger nonlinearity must reduce the imaginary part of the chemical potential. Therefore, we have to invert the movement of the intersection point $\gamma_{0}$ in the spectrum such that it is shifted to higher parameters $\gamma$ if the interaction is increased, and vice versa.

To influence the overall form of the spectrum a new parameter has to be introduced. The inversion of the movement of the intersection point can be achieved by introducing an additional asymmetry, now in the real part of the potential. Figures $1(\mathrm{c})$ and (d) show the same values as (a) and (b) but for the Hamiltonian

$$
H=\left(\begin{array}{cc}
\mathrm{i} \gamma\left(1+a_{I}\right)+a_{R} & -1 \\
-1 & -\mathrm{i} \gamma\left(1-a_{I}\right)-a_{R}
\end{array}\right),
$$

with $a_{R}=-0.15$ and $a_{I}=-0.2$.

Introducing an increased on-site energy in the loss and a decreased energy in the gain well one gets rid of the exceptional point. This can be understood intuitively since the probability densities in the two wells now differ for the ground and excited state, i.e., either the gain or the loss well is favored. However, the two previously $\mathcal{P} \mathcal{T}$-broken states and the intersection point are not lost. Instead, the ground state $\mu_{-}$turns into the upper branch, intersects with the axis $\operatorname{Im} \mu=0$, and forms a stationary state. Since the ground state has a larger probability of presence in the energetically lower gain well, its outcoupling of particles is weakened and the imaginary part of the chemical potential is shifted upwards. Consequently, the intersection point $\gamma_{0}$ for $U=0$ is shifted to a lower parameter as compared to the case $a_{R}=0$.

In this new configuration, a repulsive interaction tends to equalize both densities. This weakens the influence of the real asymmetry and shifts the imaginary part of the chemical potential down to smaller values, thus, the intersection point moves again to larger values of $\gamma$. Now, as expected, the instability due to norm oscillations vanishes and no Bogoliubov-de Gennes eigenvalue has a positive imaginary part. In fact, all eigenvalues apart from the trivial solution $\omega=0$ describing a phase shift have negative imaginary parts at the intersection point, at which the stationary state with $\operatorname{Im} \mu=0$ resides. The stationary state is therefore not only stable, but acts as an attractor. If the in- and out-coupling parameter is not at the intersection point $\gamma_{0}$, the wave function's norm grows or decays to match the appropriate interaction strength for which $\gamma_{0}=\gamma$ holds.

It is clear that the intersection point for $U=0$ is the lowest possible parameter $\gamma$ for which such an attractor can be found. If this lowest parameter is set, the wave function is attracted to the norm 0, i.e., the condensate will completely deplete. Note that due to the strong particle out-coupling the same happens if even smaller values of $\gamma$ are used. To determine this threshold, the linear wave equation is solved, and one finds that the eigenvalue of the ground state,

$$
\mu_{-}=\mathrm{i} \gamma a_{I}-\sqrt{1+\left(a_{R}+\mathrm{i} \gamma\right)^{2}},
$$

becomes real for

$$
\gamma=\frac{1-a_{R}^{2}\left(a_{I}^{-2}-1\right)}{1-a_{I}^{2}}
$$

This shows that the limit $\gamma=0$ is reached for $a_{R}=$ $a_{I} / \sqrt{1-a_{I}^{2}}$. Even stronger real asymmetries lead to a dominance of the gain contribution and therefore to a completely unstable system.

One remark has to be made on the choice of the parameters $a_{R}$ and $U$. The negative sign of the parameter $a_{R}$ leads to the intersection point of the eigenvalue of the ground state with the axis $\operatorname{Im} \mu=0$. Obviously, a positive sign of the parameter would lower the particle out-coupling of the excited state. In this case an intersection of the eigenvalue and the axis $\operatorname{Im} \mu=0$ can be achieved for attractive interaction. Therefore, it is possible to achieve real stationary states for both $U>0$ and $U<0$. However, a real ground state is only possible using repulsive interactions and $a_{R}<0$.

\section{DYNAMICS AND CONVERGENCE}

Each state of the non-Hermitian two-mode system is defined by three real parameters,

$$
\psi(R, \phi, \theta)=R\left(\begin{array}{c}
\cos (\theta / 2) \mathrm{e}^{-\mathrm{i} \phi / 2} \\
\sin (\theta / 2) \mathrm{e}^{+\mathrm{i} \phi / 2}
\end{array}\right) .
$$

Using the norm $R$ of the state as the radius, and the two angles $\theta \in[0, \pi]$ and $\phi \in[0,2 \pi)$ as spherical coordinates, every state $\psi$ is represented by a point in the three-dimensional real space.

Since the dynamics is not norm-conserving, it is not possible to restrict the discussion to the surface $R=1$, as it was possible for Graefe et al. [33] studying the dynamics of the $\mathcal{P} \mathcal{T}$-symmetric Bose-Hubbard dimer. Instead, an analysis of the complete three-dimensional dynamics as known from the $\mathcal{P} \mathcal{T}$-symmetric double-well [21] is required.

Figure 2 shows this representation for both the $\mathcal{P} \mathcal{T}$ symmetric (a) and the asymmetric case (b) with $a_{R}=$ $-0.15, a_{I}=-0.2$ for the parameters $U=1$ and $\gamma=0.7$. The dynamics of the $\mathcal{P} \mathcal{T}$-symmetric system are defined by two types of trajectories. If the wave function lies near a stable stationary point (near the south pole in Fig. 2(a)), it will start to oscillate around this point forming a closed trajectory. If it lies far away from such a stable fixed point, the wave function will follow the $\mathcal{P} \mathcal{T}$-broken state, while increasing its norm to infinity [21].

Studying Fig. 2(b) one immediately notices major differences. A dynamical attractor exists near the south pole approximately where the original stable ground state 


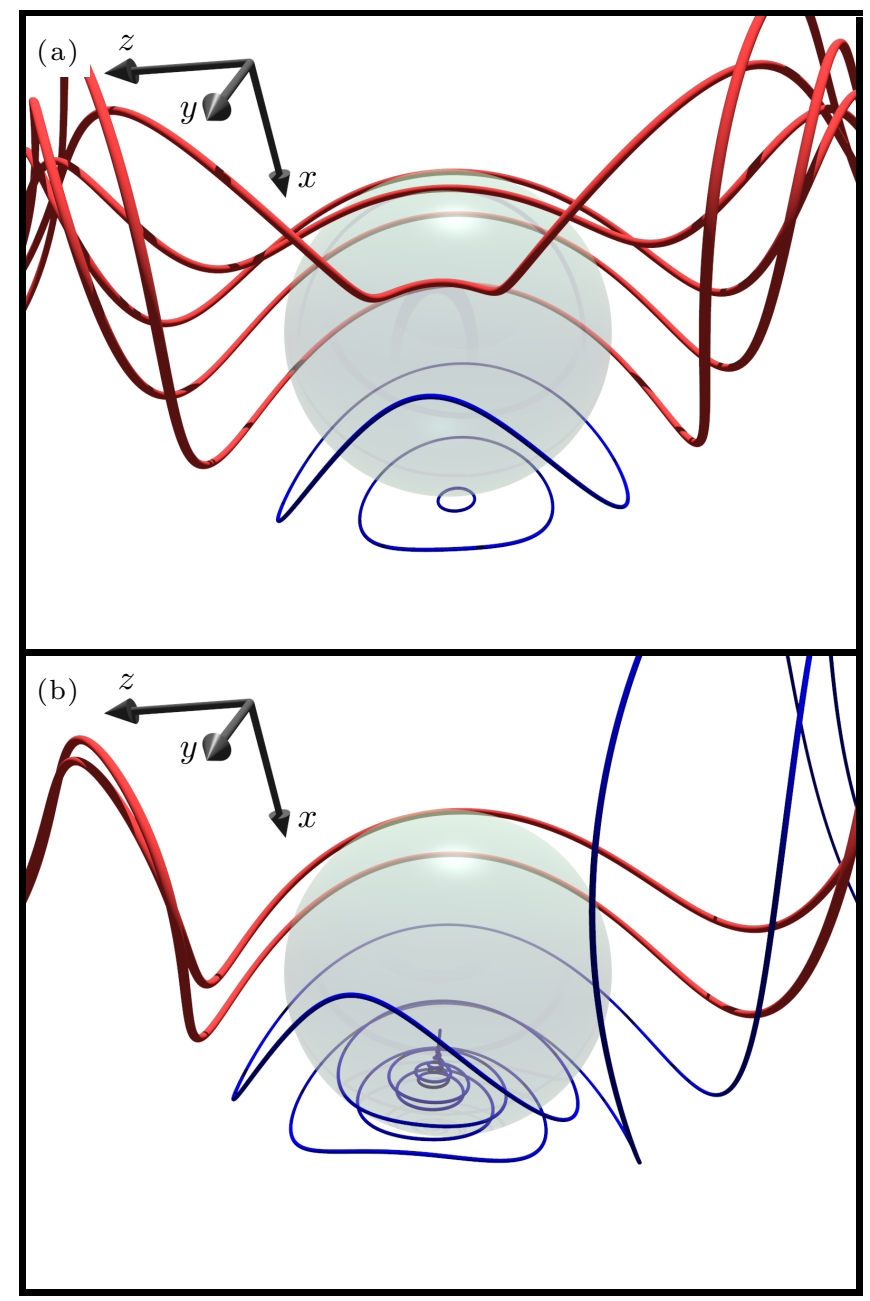

Figure 2. Bloch sphere for the parameters $U=1$ and $\gamma=0.7$. Since the radius of each state equals its norm, the transparent sphere at radius one represents all normalized states. Large $z$ values, which reside on the left side of the figure, correspond to states mainly residing in well one, i.e. the gain well, while the right side contains all states favoring the loss well. The $\mathcal{P} \mathcal{T}$-symmetric sphere (a) shows stable oscillations (thin blue closed lines), while diverging trajectories (thick red open lines) start in the loss well for $t \rightarrow-\infty$ and end up in the gain well for $t \rightarrow \infty$. All trajectories are symmetric with respect to the $x$-y-plane. In the $\mathcal{P} \mathcal{T}$-broken case (b) this symmetry is lost. While the upper two diverging trajectories (thick red lines) still run from the right to the left side, the converging trajectories (thin blue converging lines) are no longer closed.

of the $\mathcal{P} \mathcal{T}$-symmetric system resides. It lies at a norm smaller than one and at $z>0$, favoring the gain well. This is the only stable stationary point in Hilbert space and every trajectory must either converge to this point or diverge to a state with a large norm mainly localized in the gain well. Due to this convergent norm oscillations, which are one of the characteristic features of $\mathcal{P} \mathcal{T}$-symmetric systems, can only be observed for a few oscillations. The amplitude of such a norm oscillation decays until the appropriate norm, i.e., the appropriate particle number
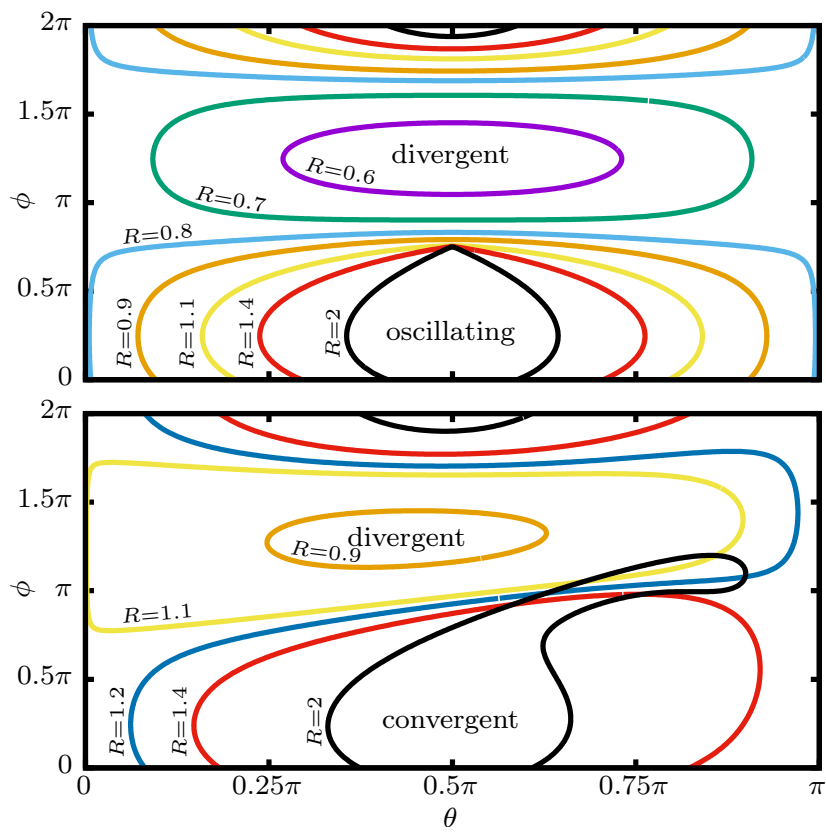

Figure 3. The intersection between the separatrix and spheres with given radii in the three-dimensional Bloch space for $U=1$ and $\gamma=0.7$. The upper panel shows the $\mathcal{P} \mathcal{T}$-symmetric case with $a_{R}=0$ and $a_{I}=0$ where the separatrix divides the space between stable oscillations and diverging wave functions. The lower panel presents the asymmetric case with $a_{R}=-0.15$ and $a_{I}=-0.2$; due to the attractor, no oscillating trajectories can be found and all wave functions are either divergent or convergent. In both cases, the separatrix reaches the lowest radii for $\theta \approx 0.5 \pi$ and $\phi \approx 1.25 \pi$. For higher radii, more wave functions become divergent, until only a small region around $\theta \approx 0.5 \pi$ and $\phi \approx 0.25 \pi$ remains stable. The two cases differ in two aspects: The asymmetric case supports convergent wave functions with higher norms and is not symmetric to the axis $\theta=0.5 \pi$ like the $\mathcal{P} \mathcal{T}$-symmetric situation.

of the stationary state, is reached.

It is apparent that the convergent area of the asymmetric system is similar to the area of stable oscillations in the $\mathcal{P} \mathcal{T}$-symmetric case. The only qualitative difference between these two cases lies in the fact that the region of the $\mathcal{P} \mathcal{T}$-symmetric case is closed in positive and negative $z$-direction, while its counterpart with $a_{R}=-0.15$ and $a_{I}=-0.2$ includes strongly asymmetric states with large norms from the loss well. The exact separatrix between the divergent and the attractive region can be calculated in a straightforward manner, however, a three dimensional presentation as done in Fig. 2 does not allow a quantitative analysis. Instead, it is beneficial to restrict the calculation to discrete interaction strengths, i.e., discrete radii in the Bloch representation, which can be characterized as either convergent or divergent. The results are shown in Fig. 3, in which the $\mathcal{P} \mathcal{T}$-symmetric and the asymmetric case are compared. In both cases, the first configurations that start to diverge are evenly distributed between both wells and possess currents from the loss to 
the gain well due to the phase difference $\phi \approx 1.25 \pi$. At this point the first important difference becomes apparent. While the $\mathcal{P} \mathcal{T}$-symmetric case becomes unstable at $R \approx 0.6$, the asymmetric case does not show any diverging trajectories up to $R \approx 0.85$. For larger radii the divergent region grows quickly, symmetric around $\theta=0.5 \pi$ for the $\mathcal{P} \mathcal{T}$-symmetric case and strongly asymmetric for the case $a_{R}=-0.15$ and $a_{I}=-0.2$. The asymmetry is the second important difference between both cases. This is essential for large radii, as the case $R=2$ demonstrates in Fig. 3 . Due to the broken symmetry wave functions with a major occupation in the loss well are able to converge to the attractor.

\section{EXTENDED POTENTIALS}

In the previous sections we discussed a two-mode system which describes a Bose-Einstein condensate with asymmetric gain and loss of particles. For a more quantitative analysis the one-dimensional Gross-Pitaevskii equation is solved numerically exact without the restriction to a finite set of basis vectors. The Gross-Pitaevskii equation reads

$$
\left(-\partial_{x}^{2}+V(x)+g|\psi(x, t)|^{2}\right) \psi(x, t)=\mathrm{i} \partial_{t} \psi(x, t)
$$

with the strength of the nonlinear contact interaction $g$ and the asymmetric complex double-well potential

$$
V(x)=\left\{\begin{array}{ll}
V_{1}(x), & x \leq 0 \\
V_{2}(x), & x>0
\end{array} .\right.
$$

The left half of the potential, $x \leq 0$, is chosen as

$$
V_{1}(x)=\frac{1}{4} x^{2}+4 e^{-\frac{1}{2} x^{2}}-i \gamma x e^{-0.12 x^{2}} .
$$

It describes a single well composed of a harmonic trapping potential and a Gaussian barrier at $x=0$.

The imaginary part is positive and thus describes particle gain. Its strength is controlled by the parameter $\gamma$. The right well, $x>0$, is modified,

$$
V_{2}(x)=\frac{1}{4} x^{2}+4 e^{-\left(\frac{1}{2}+a_{R}\right) x^{2}}-i \gamma x e^{\left(-0.12+a_{I}\right) x^{2}} .
$$

In this well, particle loss is applied which is weakened for $a_{I}>0$ and strengthened for $-0.12<a_{I}<0$. The parameter $a_{R}$ shrinks or expands the barrier, i.e., lowers or raises the potential in the loss well. Note that, as in the two-mode system, negative values of $a_{R}$ and $a_{I}$ correspond to a stronger particle loss and a shallower loss well. For the case $a_{R}=a_{I}=0$, both halves become equal and result in an extensively studied $\mathcal{P} \mathcal{T}$-symmetric potential [19-21]. The potential is shown in Fig. 4 for various parameters $a_{R}$ and $a_{I}$.

First we study whether and where stable stationary states can be found for non-vanishing asymmetries. Numerical calculations show that the conclusions from the
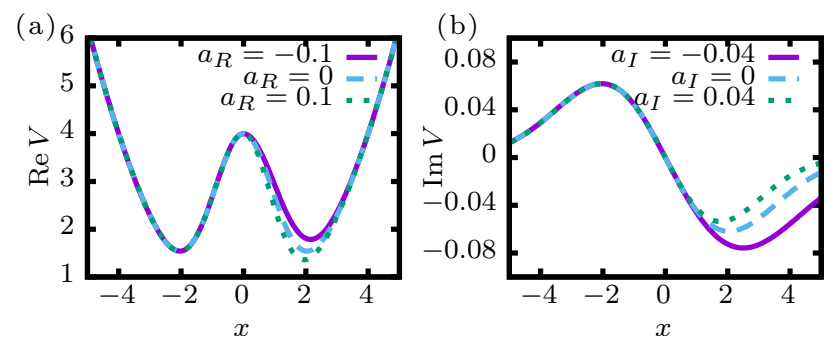

Figure 4. Real part (a) and imaginary part (b) of the spatially extended potential (11) and (10) for various asymmetry parameters and $\gamma=0.05$.
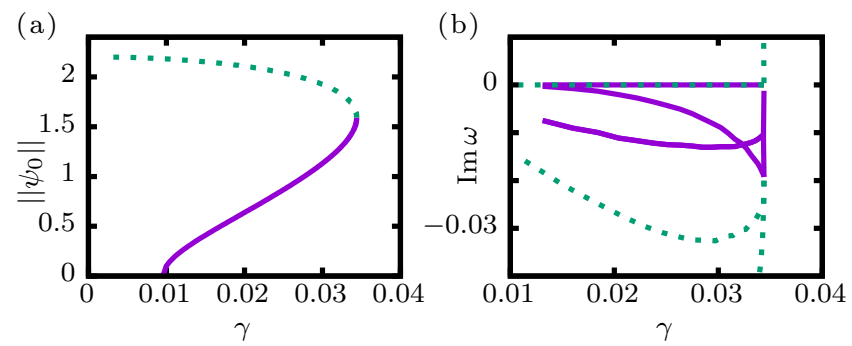

Figure 5. Norm of the stationary states (a) and their four smallest Bogoliubov-de Gennes eigenvalues (b) over the value of the in- and out-coupling parameter $\gamma$ for $g=0.1, a_{R}=$ -0.01 and $a_{I}=-0.08$. As long as larger values of $\gamma$ support a higher norm of the stationary state (solid line), it is stable. Therefore, the upper branch (dotted line) is unstable for all parameters $\gamma$.

two-mode system hold true also for the spatially extended potential. The ground state shows the desired behavior for repulsive interactions and a stronger particle loss if, at the same time, the on-site energy in the loss well is increased. We therefore set the parameters to $a_{R}=-0.01$, $a_{I}=-0.08$, and $g=0.1$. The parameters used correspond to a relative imbalance of the well's depths of about $1 \%$ and an imbalance of the particle in- and out-coupling of about $25 \%$. Note that, compared to the experiment [28], the interaction strength is very weak, i.e., Feshbach resonances [34] would have to be employed. The stationary state for each parameter $\gamma$ is calculated and its norm, i.e., the necessary nonlinear strength to stabilize this in- and out-coupling, is shown in Fig. 5(a). For most parameters $\gamma$ two stationary states can be found. The lower state in Fig. 5(a) has a higher stable norm for larger values of $\gamma$, thus, stabilizing itself against norm oscillations as discussed previously for the two-mode model. The second branch results from the fact that stronger interaction strengths shift the stationary state back to smaller parameters $\gamma$. This was shown in Fig. 1(c). However, in this range the state is not protected against norm oscillations, and therefore is unstable.

The Bogoliubov-de Gennes eigenvalues shown in Fig. 5(b) confirm these considerations. There are three different types of perturbations, one of which is the trivial solution with $\operatorname{Im} \omega=0$. The second type becomes zero for 
a vanishing norm. Additional studies of the data confirm that the eigenvalue is approximately linear in the squared norm of the stationary state and negative. This is exactly the behavior we expect from a stable perturbation of the wave function's norm and is only found for the lower branch. The eigenvalues of the third type have a finite value even for a vanishing norm. At this point, the particle-particle interaction vanishes, i.e., the perturbation exists even for linear systems. It corresponds to a higher excited state which decays exponentially since the particle out-coupling is stronger than the in-coupling into the system. Even though only one of them is shown in Fig. 5, all such eigenvalues are negative, i.e., they correspond to stable solutions.

To put the quantitative results into perspective, we shortly remind of the corresponding results from the $\mathcal{P} \mathcal{T}$ symmetric system [19]. For small parameters $\gamma$, this system supports two stationary states for a wide range of interaction strengths. The $\mathcal{P} \mathcal{T}$ symmetry breaking occurs at $\gamma \approx 0.042$ after which the $\mathcal{P} \mathcal{T}$-symmetric stationary states vanish. Figure 5 shows that the range of stable stationary ground states is $0.01 \lesssim \gamma \lesssim 0.035$, i.e., it includes most of the original range in the $\mathcal{P} \mathcal{T}$-symmetric system.

As a final test the attractive behavior of the stationary state is examined in dynamical calculations. To reduce the number of parameters describing the time-dependent wave function, the Bloch-sphere representation is used again. Since the Hilbert space is not two-dimensional anymore, we choose a projection onto the space spanned by the normalized ground state $e_{1}=\psi_{g}$ and the orthogonal vector $e_{2}=\alpha\left(\psi_{e}-\left\langle\psi_{g}, \psi_{e}\right\rangle \psi_{g}\right)$ selected by the GramSchmidt method, where $\alpha$ is the normalization constant. Calculations from [21] for the $\mathcal{P} \mathcal{T}$-symmetric case show that this is a good approximation for the system considered. An arbitrary state in this basis can be written as in Eq. (7) and is therefore defined by the three real parameters $R, \phi$ and $\theta$. Note that the orthonormal twodimensional basis is different from the choice made in the two-mode approximation and, thus, the parameters $\phi$ and $\theta$ must differ.

We study two distinct cases which differ only in the strength of the in- and out-coupling parameter $\gamma_{1} \approx$ 0.0277 and $\gamma_{2} \approx 0.0366$. Both cases support a stationary state $\psi_{i}$ with norms $R_{1}=1$ and $R_{2}=\sqrt{2}$ and the two Bloch angles $\phi_{i}$ and $\theta_{i}$. Figure 6 shows the time evolution of the three Bloch coordinates for both states in both systems. We see that the coordinates $R_{i}, \phi_{i}, \theta_{i}$ of both states converge to the appropriate dynamical attractor, i.e., they converge to the coordinates of $\psi_{1}$ in the left panel and of $\psi_{2}$ in the right panel. The time of the convergence to the stationary states differs in the two cases. This results from the difference of the smallest Bogoliubov-de Gennes eigenvalue which is inversely proportional to the timescale of the convergence. Here $|\operatorname{Im} \omega|$ from $\psi_{2}$ is larger than $\psi_{1}$, therefore, the time of convergence for $\psi_{1}$ is smaller. This is confirmed by Fig. 6. Thus, we have shown that the behavior of the two-mode system is also

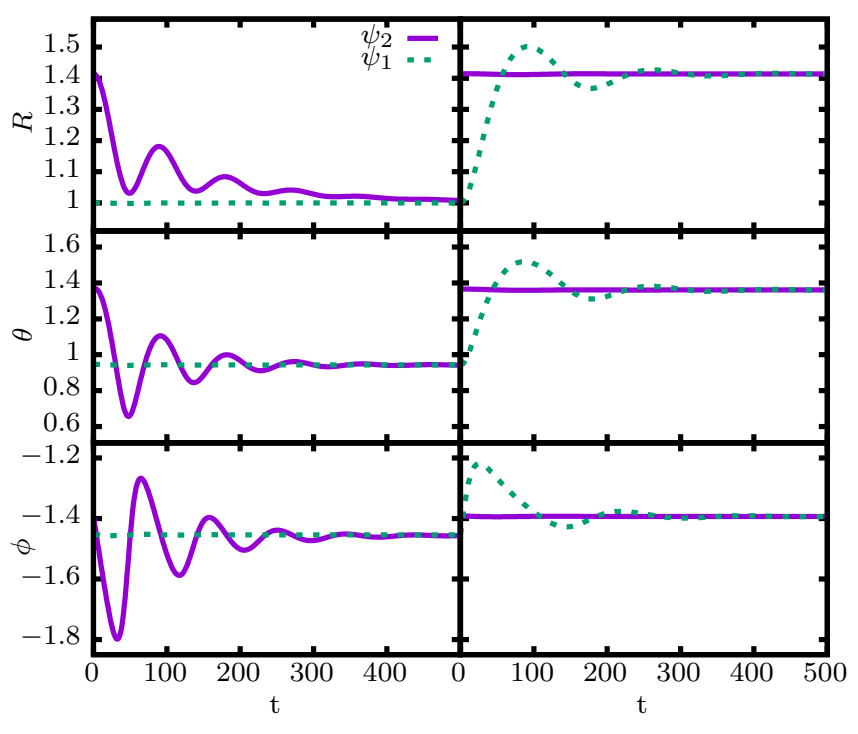

Figure 6. Time evolution of the Bloch coordinates of the two stationary states $\psi_{i}$ for $\gamma_{1} \approx 0.0277$ (green dotted line) and $\gamma_{2} \approx 0.0366$ (magenta solid line). In the left panel a system with $g=0.1, a_{R}=-0.01, a_{I}=-0.08$, and $\gamma \approx 0.0277$ is used. Hence, $\psi_{1}$ is stationary while $\psi_{2}$ converges to $\psi_{1}$ within approximately four oscillations. In the right panel the in- and out-coupling parameter is changed to $\gamma \approx 0.0366$, and the two states exchange their roles.

found in a realistic spatially extended system, i.e., for a large range of values of the coupling strength $\gamma$, the stationary states act as attractors.

\section{CONCLUSION}

In this work we studied Bose-Einstein condensates in an asymmetric non-Hermitian double well. In an experiment such asymmetries are always unavoidable. However, we were able to show that it is possible to manipulate the system in such a way that an attractor of the dynamics exists which possesses all properties of an $\mathcal{P} \mathcal{T}$-symmetric state required to identify it in an experiment. First, we presented stationary solutions to the Gross-Pitaevskii equation of a two-mode system with asymmetric gain and loss. One requirement for a stable realization can be formulated a priori: If the particle gain is stronger than the particle loss, perturbations with high excitation energies will be exponentially enhanced rendering the state unstable. Therefore, the particle loss must always be stronger than the gain. In this configuration, the ground state of the system cannot only be made stable but becomes a dynamical attractor. To achieve this, a real asymmetry of the trapping potential was introduced, reducing the particle density of the ground state in the loss well, i.e., the stronger particle loss is partially counterbalanced by the asymmetric trap.

Next, the dynamical properties were carefully studied 
using a specific asymmetric potential and a fixed repulsive contact interaction. Weak asymmetries leave the $\mathcal{P} \mathcal{T}$-symmetric oscillations mainly intact. However, all such wave functions end up at the dynamical attractor, effectively limiting the timescale during which such oscillations can be observed. It was shown that the convergent region is indeed even larger than in the $\mathcal{P} \mathcal{T}$-symmetric case including a set of wave functions with large norms residing in the loss well.
Finally, the results were compared to those of a realistic spatially extended potential. Not only do all observations from the two-mode approximation remain valid, but in addition we were able to show that an attractor exists in a wide range of particle in- and out-coupling strengths. Therefore, the potential is capable of acting as a setup for a $\mathcal{P} \mathcal{T}$-symmetric realization with unbalanced gain and loss.
[1] C. M. Bender and S. Boettcher, Phys. Rev. Lett. 80, 5243 (1998).

[2] C. M. Bender, D. C. Brody, and H. F. Jones, Phys. Rev. Lett. 89, 270401 (2002).

[3] A. Mostafazadeh, J. Phys. A 41, 055304 (2008).

[4] A. Mostafazadeh, Int. J. Geom. Methods Mod. Phys. 07, 1191 (2010).

[5] R. El-Ganainy, K. G. Makris, D. N. Christodoulides, and Z. H. Musslimani, Opt. Lett. 32, 2632 (2007).

[6] S. Klaiman, U. Günther, and N. Moiseyev, Phys. Rev. Lett. 101, 080402 (2008).

[7] Z. H. Musslimani, K. G. Makris, R. El-Ganainy, and D. N. Christodoulides, Phys. Rev. Lett. 100, 030402 (2008).

[8] K. G. Makris, R. El-Ganainy, D. N. Christodoulides, and Z. H. Musslimani, Phys. Rev. Lett. 100, 103904 (2008).

[9] K. G. Makris, R. El-Ganainy, D. N. Christodoulides, and Z. H. Musslimani, Phys. Rev. A 81, 063807 (2010).

[10] A. Guo, G. J. Salamo, D. Duchesne, R. Morandotti, M. Volatier-Ravat, V. Aimez, G. A. Siviloglou, and D. N. Christodoulides, Phys. Rev. Lett. 103, 093902 (2009).

[11] C. E. Rüter, K. G. Makris, R. El-Ganainy, D. N. Christodoulides, M. Segev, and D. Kip, Nat. Phys. 6, 192 (2010).

[12] B. Peng, Ş. K. Özdemir, F. Lei, F. Monifi, M. Gianfreda, G. L. Long, S. Fan, F. Nori, C. M. Bender, and L. Yang, Nat. Phys. 10, 394 (2014).

[13] A. Regensburger, C. Bersch, M.-A. Miri, G. Onishchukov, D. N. Christodoulides, and U. Peschel, Nature 488, 167 (2012).

[14] J. Schindler, A. Li, M. C. Zheng, F. M. Ellis, and T. Kottos, Phys. Rev. A 84, 040101 (2011).

[15] N. Bender, S. Factor, J. D. Bodyfelt, H. Ramezani, D. N. Christodoulides, F. M. Ellis, and T. Kottos, Phys. Rev. Lett. 110, 234101 (2013).

[16] J. Li, A. K. Harter, J. Liu, L. de Melo, Y. N. Joglekar, and L. Luo, "Observation of parity-time symmetry breaking transitions in a dissipative floquet system of ultracold atoms," (2016), arXiv:1608.05061 [cond-mat.quant-gas].

[17] H. Cartarius, D. Haag, D. Dast, and G. Wunner, J. Phys. A 45, 444008 (2012).

[18] H. Cartarius and G. Wunner, Phys. Rev. A 86, 013612 (2012).

[19] D. Dast, D. Haag, H. Cartarius, G. Wunner, R. Eichler, and J. Main, Fortschr. Physik 61, 124 (2013).

[20] D. Dast, D. Haag, H. Cartarius, J. Main, and G. Wunner, J. Phys. A 46, 375301 (2013).

[21] D. Haag, D. Dast, A. Löhle, H. Cartarius, J. Main, and G. Wunner, Phys. Rev. A 89, 023601 (2014).

[22] M. Kreibich, J. Main, H. Cartarius, and G. Wunner, Phys. Rev. A 87, 051601(R) (2013).

[23] F. Single, H. Cartarius, G. Wunner, and J. Main, Phys. Rev. A 90, 042123 (2014).

[24] T. Gericke, P. Wurtz, D. Reitz, T. Langen, and H. Ott, Nat. Phys. 4, 949 (2008).

[25] N. P. Robins, C. Figl, M. Jeppesen, G. R. Dennis, and J. D. Close, Nat. Phys. 4, 731 (2008).

[26] F. Cannata, G. Junker, and J. Trost, Phys. Lett. A 246, 219 (1998).

[27] B. Bagchi and C. Quesne, Phys. Lett. A 273, 285 (2000).

[28] R. Gati and M. K. Oberthaler, J. Phys. B 40, R61 (2007).

[29] G. Barontini, R. Labouvie, F. Stubenrauch, A. Vogler, V. Guarrera, and H. Ott, Phys. Rev. Lett. 110, 035302 (2013).

[30] E.-M. Graefe, J. Phys. A 45, 444015 (2012).

[31] I. Bloch, J. Phys. B 38, S629 (2005).

[32] P.-G. de Gennes, Superconductivity of Metals and Alloys, reprint ed. (Addison-Wesley, Redwood City, 1989).

[33] E. M. Graefe, H. J. Korsch, and A. E. Niederle, Phys. Rev. Lett. 101, 150408 (2008).

[34] S. Inouye, M. R. Andrews, J. Stenger, H.-J. Miesner, D. M. Stamper-Kurn, and W. Ketterle, Nature 392, 151 (1998). 